TITRE: LIRE POUR ÉCRIRE. L'ÉCRITURE MIROIR DE LA LECTURE

Auteur(s): MARIn Brigitte, Professeure des universités, UniVersité Paris-EST Créteil (UPEC) - ESPE

Publication: PeRSPECTIVES ACTUELles SUR L'APPRENTISSAGE DE LA LECTURE ET DE L'ÉCRITURE/ CONTRIBUTIONS ABOUT LEARNING TO READ AND WRITE - ACTES DU SYMPOSIUM INTERNATIONAL SUR LA LITÉRACIE À L'ÉCOLE/INTERNATIONAL SYMPOSIUM FOR EDUCATIONAL LITERACY (SILE/ISEL) 2015

PAGES: $120-140$

Directeurs: Marie-France Morin, Denis Alamargot et Carolina Gonçalves.

ÉditeUR: LES ÉdITIONS DE L'UNIVERSITÉ DE SHERBROOKE, 2016.

ISBN: 978-2-7622-0355-4

URI: HTTP://HDL.HANDLE.NET/11143/10232

DOI: HTTPS://DOI.ORG/10.17118/11143/10232 


\section{Lire pour écrire. L'écriture miroir de la lecture}

Marin Brigitte

Professeure des universités, Université Paris-Est Créteil (UPEC) - ESPE

Résumé: Cette étude a pour objectif de mettre en évidence l'effet de ressources lexicales ciblées, sur l'évolution des compétences d'écriture narrative d'élèves de l'école élémentaire française. Les performances de 150 élèves ont été mesurées pour comparer les résultats d'un groupe témoin et d'un groupe expérimental. L'évolution de la longueur des textes produits, entre le prétest et le posttest, a été analysée. En parallèle, la comparaison des versions 1 et 2 de textes rédigés par ces élèves a permis d'établir les caractéristiques des ajouts portés sur un texte écrit, puis révisé, en fonction du statut du groupe d'appartenance (groupe témoin vs groupe expérimental). Les analyses quantitatives et qualitatives indiquent que l'exposition régulière à des dispositifs didactiques fondés sur une conception raisonnée de l'acquisition lexicale permet simultanément de faire progresser les élèves dans la maîtrise du vocabulaire et des habiletés littéraciées.

Mots clés : Écriture - Vocabulaire - Littéracie - Compétences - Textes narratifs

Abstract: This study aims at highlighting the effect of targeted lexical resources, on the evolution of the skills of pupils' narratives at French elementary school. The performances of 150 pupils were measured to compare the results of a witness group and of an experimental group. The evolution of the length of the texts was analyzed between the pretest and the posttest. In parallel, the comparison of the versions 1 and 2 of texts, written, then revised by these pupils allowed to establish the characteristics of the words added to the first draft of the text, according to the status of the membership group (witness group vs experimental group). The quantitative and qualitative analyses indicate that the regular exposure to didactic devices based on a rational conception of lexical apprenticeship helps students to improve both their vocabulary and their literacy skills.

Key words: Writing - Vocabulary - Literacy - Skills - Narratives 


\section{Introduction}

De récentes orientations de la recherche sur l'écriture à l'école élémentaire ont mis en évidence le rôle des dispositifs pédagogiques dans l'amélioration des compétences rédactionnelles des élèves. Cette question est particulièrement d'actualité dans un contexte plus général où le lien entre recherche et formation suppose le transfert des données issues de la recherche pour faire évoluer les manières de faire la classe des enseignants en vue de la réussite du plus grand nombre d'élèves. Devant un tel défi, le partage de problématiques communes aux chercheurs et enseignants s'avère nécessaire pour reconfigurer les questions professionnelles en questions de recherche et assurer la circulation des résultats entre deux univers jusque-là trop cloisonnés et éloignés.

Depuis les recherches appliquées - à l'enseignement - jusqu'aux actuelles recherches impliquées - de l'ensemble de tous les acteurs de la formation et de l'enseignement - une nouvelle ère s'ouvre dans les partenariats entre praticiens et chercheurs concernés par le domaine de l'enseignement et des apprentissages. La recherche dont les premiers résultats sont donnés dans cet article s'inscrit dans une démarche de recherche impliquée, avec l'étroite collaboration d'un enseignant-chercheur et d'un maître-formateur acculturé à la recherche, en lien avec les enseignants des classes qui ont, d'une part, accepté de participer au dispositif, et d'autre part, montré leur intérêt pour la conception du protocole de recherche et ses résultats. Dans ce registre, elle relève plus précisément d'une recherche pour l'éducation au sens où elle vise à améliorer directement les pratiques enseignantes au bénéfice des élèves, avec une dissémination à très court terme (en phase avec le dispositif conçu en contexte écologique avec les enseignants des classes) et à moyen terme pour la communauté d'enseignants.

L'évolution terminologique des expressions utilisées pour désigner la recherche en éducation est en effet éloquente, avec un jeu de variation des prépositions utilisées pour caractériser ces recherches, qui deviennent tour à tour recherches en éducation, mais aussi, sur l'éducation, pour l'éducation, ou encore autour de l'éducation. Ainsi la recherche en éducation cible-t-elle un point d'ancrage, une thématique globale, alors que la recherche sur l'éducation concerne un objet. Le vocable de recherche pour l'éducation se fonde, quant à lui, sur la production de résultats. Plus largement, la recherche autour de l'éducation requiert la mobilisation de disciplines différentes, parfois hétérogènes dans leur méthodologie et leur épistémologie, au service de l'étude et de l'amélioration des apprentissages des élèves. Ce sont autant de changements de points de vue et d'objectifs en œuvre pour délimiter le périmètre attendu d'études destinées à renforcer le lien entre les résultats qu'elles produisent et leurs retombées dans les classes. C'est le cas du volet de la recherche qui est présenté.

Les new literacy studies (Barton et Hamilton, 2010) ont remis sur le devant de la scène la question de la littératie qui constitue une préoccupation majeure des enseignants, avec une focalisation particulière sur l'exercice de l'écrit. Rarement enseigné formellement, l'écrit mérite une attention

1. Annick Cautela, maître-formateur, Université Paris-Est Créteil - ESPE de l'académie de Créteil. 
particulière, d'autant qu'il est un sérieux prédicteur de réussite ou d'échec scolaire et le véhicule privilégié de la restitution, comme de l'évaluation des savoirs. À l'école élémentaire, la chronologie de l'apprentissage de l'écriture et le temps qui lui est consacré accordent la première place à l'écrit narratif (Garcia-Debanc, Duvignau, Dutrait et Gangneux, 2009) dont la réalisation est souvent perçue comme un allant-de-soi, ne supposant pas nécessairement d'enseignement spécifique. C'est par ailleurs une demande de formation récurrente des enseignants de l'école primaire que d'avoir accès à des ressources sur ces besoins prioritaires.

Le choix de l'école élémentaire comme terrain de recherche sur cet objet est dicté par une contrainte pragmatique. En effet, si les enseignants de collège et a fortiori de lycée orientent leurs évaluations selon une logique normative, destinée à préparer aux examens certificatifs, les professeurs des écoles visent plus couramment le principe d'évaluation formative. L'analyse des performances des élèves obéit plus volontiers à une logique d'étayage à partir des erreurs relevées.

Le but de l'étude, axée sur la didactique de l'écrit, est de préciser le rôle de pratiques d'enseignement du lexique dans l'évolution des compétences d'écriture d'élèves de la troisième année de l'école élémentaire, dès lors qu'elles fournissent des matériaux et des méthodes susceptibles de les aider à écrire des textes narratifs. Nous nous intéressons en particulier à l'usage du lexique pour envisager la manière dont celui-ci peut devenir un catalyseur de l'activité d'écriture. La question de recherche centrale porte sur les effets de dispositifs didactiques permettant de stabiliser à moyen et à long terme les connaissances lexicales pour les réinvestir en discours.

\section{Cadre théorique}

L'activité rédactionnelle est une activité particulièrement complexe dans la mesure où elle mobilise différents régimes de compétences, qu'elles concernent l'écriture ou la réécriture, en temps réel, avec correction au fil du texte ou différée lors de la phase de récriture a posteriori. Elle a un coût cognitif important, variable selon l'expertise du scripteur (McCutchen, 2011), et dans laquelle entre la maîtrise du vocabulaire lié au domaine concerné par le texte à écrire.

Les travaux récents sur le vocabulaire à l'école élémentaire ont montré l'écart entre le vocabulaire scolaire réellement connu des élèves et celui supposé acquis par les programmes et les manuels scolaires. Une corrélation a été établie entre la maîtrise insuffisante du vocabulaire requis et l'échec scolaire (Lee, 2011), notamment dans les textes informatifs à visée scientifique où la méconnaissance du lexique disciplinaire peut se traduire par des attitudes d'abstention face à l'écrit (Marin, 2013). De manière moins visible, mais tout aussi disqualifiante, le texte narratif, censé reproduire les situations de la vie quotidienne ou des modèles de fictions, requiert cependant une maîtrise rhétorique indissociable d'un panel lexical diversifié, que ne saurait épuiser la seule désignation mimétique des faits et des personnages du récit. L'élaboration de scenarios développés suppose une fluence lexicale permettant de traduire la complexité de la perception des actions et des intentions des personnages 
mis en scène dans le récit pour traduire la causalité du monde physique et la causalité intentionnelle. La théorie de l'intentionnalité prélinguistique (Searle, 2012) invite à poser le langage comme vecteur de la formalisation de schèmes narratifs majeurs préexistant à leur traduction en mots.

À partir des perceptions et des actions, [on est] capable d'élaborer des souvenirs et des intentions premières aussi bien que des croyances et des désirs ou encore d'autres formes d'intentionnalité comme l'espoir et la crainte, la colère et l'agressivité.[...] Nous devrions voir les fondements biologiques du langage dans l'intentionalité prélinguistique. (Searle, 2012, p. 231).

Le rôle des activités lexicales permettant d'augmenter le vocabulaire actif des élèves de l'école élémentaire est ainsi déterminant dans le développement de leurs compétences rédactionnelles, incluant la mise en scène des chaînes de causalité.

L'activité d'écriture du lecteur novice est rendue plus difficile dès lors que le vocabulaire attendu n'est pas immédiatement accessible en mémoire. Or, le processus d'acquisition du vocabulaire est directement lié à la construction en mémoire sémantique des représentations des signifiés auxquelles sont associés des signifiants (Denhière et Jhean-Larose, 2011). La permanence de ces représentations dépend des phénomènes mnésiques, dont la propension à l'oubli et, par conséquent, à la nécessité de réactivation qui s’opère, notamment par la verbalisation (Rosenthal et Ehri, 2011) et la structuration du vocabulaire (Verhoeven, 2011). Les activités de catégorisation et de hiérarchisation, en évitant de surcharger la mémoire de travail, favorisent l'encodage et la compréhension.

Parmi les incontournables de la rédaction de fiction figure l'appréhension de la typicalité ${ }^{2}$ qui contribue à programmer les acquisitions (Denhière et Jhean-Larose, 2011), la capacité à distinguer le spécifique du générique, laquelle suppose de disposer de réseaux sémantiques suffisamment construits pour percevoir, par exemple, la hiérarchie entre hyponymes et hyperonymes.

Le développement du lexique s’organise selon plusieurs degrés de complexité. Au stade élémentaire, l'apprentissage des termes référentiels répond au principe de la monstration et correspond en grande partie à la catégorie du nom, lequel se prête à un déploiement du vocabulaire en extension, dans une perspective taxinomique, voire encyclopédique. Dans les activités d'apprentissage lexical, la surreprésentation du nom au détriment d'autres catégories grammaticales renvoie à « une conception scolaire spontanée de la signification comme rapport référentiel direct, de désignation par rapport à des entités du monde : le mot renvoie à une chose, correspond à une réalité stable, prédécoupée et identifiable dans le monde» (Nonnon, 2011, p. 3).

Lors de la découverte du vocabulaire, la conscience morphologique permet d'accéder au sens des mots (Ducan, Colé et Casalis, 2009; Reed, 2008). Ainsi la morphologie dérivationnelle favorise-t-elle la compréhension et la mémorisation de mots complexes identifiés à partir des mots simples dont

2. Cependant, tous les exemplaires d’une même catégorie ne sont pas également représentatifs de cette catégorie. 
ils sont issus. La compréhension des mots complexes demande un traitement sémantique et syntaxique approfondi (Colé, 2011), lequel est facilité par une structure phonologique et sémantique transparente. L'acquisition de la signification relève d'une relation de désignation, mais nécessite parfois une réorganisation de l'expérience et des significations de mots déjà en usage. Les termes correspondant à une notion intégratrice supposent une élaboration conceptuelle exigeante sur le plan cognitif. L'appréhension de ce degré de complexité suppose d'apprécier ces différences dans les modes d’élaboration de la référence et de la signification (Nonnon, 2012).

Les activités orales d'encodage de la pensée se fondent sur la sensibilisation à la paraphrase (Jousse, Polguère et Tremblay, 2008), comme sur le recours à la synonymie (Kleiber, 2009). Par ailleurs, l'interaction entre écriture et apprentissage lexical a maintes fois été mise en évidence à partir d'études de terrain. La production écrite apparaît comme un élément déterminant de l'appropriation lexicale, notamment au regard d'interactions verbales fondées sur des activités de l'écrit (Sardier, 2012) qui engendrent la pensée et « le surgissement des mots sous la plume» (Bucheton, 2014, p. 178).

La structuration du vocabulaire permet l'accès à la catégorisation et à la mémorisation des mots, en contexte et hors contexte. Elle est l'un des déclencheurs du processus d'écriture et/ou de récriture; elle favorise la densification du récit (Garcia-Debanc et al. (2009) et permet de l'agrémenter en augmentant les circonstances et les péripéties qui lui donnent vie. Sur le plan textuel, la progression de l'usage lexical est consubstantiellement liée aux progrès rédactionnels (Sardier et Grossmann, 2010), l'un et l'autre processus, de développement des connaissances lexicales et du processus d'écriture, interagissant pour s'alimenter mutuellement.

La dimension écologique de la recherche - dont sont exposés infra les premiers résultats - prend en compte les dimensions protéiformes du processus d'évaluation en permettant à l'enseignant d'identifier les difficultés des élèves pour y remédier (Wanlin et Crahay, 2012).La dimension de transfert des résultats de la recherche conduit à détecter les indices à l'origine de la production d'indicateurs caractérisant les obstacles à la réalisation des tâches prescrites en vue d'apporter une aide à l'élève (Dechamboux et Mottier-Lopez, 2015). C'est au niveau de cette prise d'indices à partir des productions d'élèves que s'est effectué le travail de constitution du corpus et de recueil de données.

\section{Méthode}

Cette étude teste un dispositif d'aide à l'écriture et à la réécriture de textes narratifs fondé sur la mise à disposition d'aides lexicales. Ces aides ont été élaborées en cohérence thématique avec les textes que les élèves ont eu à rédiger. Elles concernent, d'une part, l'aspect linguistique de l'apprentissage lexical, en développant un axe paradigmatique et un axe syntagmatique pour structurer cet apprentissage. Elles sont ainsi catégorisées à partir de deux critères, la nature grammaticale et narratologique des aides proposées. Elles portent d'autre part sur l'aspect langagier et le développement des habiletés littéraciées par la mise à disposition de ressources lexicales, non plus seulement en 
langue, mais en discours, par des textes de littérature de jeunesse portant sur les mêmes « figures littéraires » (Crinon, Marin et Lallias, 2006) que les textes lanceurs de l'écriture.

La question de recherche centrale concerne les effets d'un dispositif didactique permettant de stabiliser à moyen et à long terme des connaissances lexicales pour les réinvestir en discours, en situation d'écriture narrative. Elle porte sur l'effet de telles aides contextuelles et plus largement sur le rôle du vocabulaire actif et passif lors d'activités d'écriture susceptibles de le mobiliser. La réflexion porte sur la mobilisation en situation des mots étudiés et la construction de ressources pour la formation.

\section{Participants}

Pour le volet de la recherche envisagé dans le cadre de cette étude, qui porte globalement sur 423 élèves de troisième, quatrième et cinquième années de l'école élémentaire, nous nous sommes focalisée plus particulièrement sur le niveau de classe de troisième année. Les participants retenus sont au nombre de 150 (77 filles et 73 garçons) (cf. Tableau 1), scolarisés dans quatre écoles de la banlieue est-parisienne, situées en France, dans deux départements géographiques ${ }^{3}$ de l'académie de Créteil. Les sujets de l'étude ont été sélectionnés dans les huit classes qui ont participé à l'expérimentation, se répartissant en un groupe expérimental et un groupe témoin.

Les groupes ont été égalisés de manière à pouvoir comparer les résultats des groupes expérimentaux et des groupes témoins. En fonction de la réalité des publics qui composent les classes de cette académie, nous avons conservé, pour l'étude dont nous restituons ici les premiers résultats, les élèves francophones afin de ne pas introduire le biais de la connaissance de la langue. Notre échantillon a été divisé en deux groupes en fonction de l'appartenance au groupe expérimental ou au groupe témoin : groupe témoin ; n = 62 ; groupe expérimental ; n = 88 (cf. Tableau 1).

Nous avons établi les échantillons d'après la manière dont le lexique pour écrire a fait (ou n’a pas fait) l'objet d'un enseignement explicite au cours des années antérieures afin de prendre en compte des habitudes de sélection lexicale comparables. Dans l'ensemble des écoles participant à la recherche, aucune stratégie particulière d'enseignement du vocabulaire en lien avec l'écriture de textes narratifs n’avait été mise en place.

Les catégories socioprofessionnelles des parents sont assez homogènes en fonction de la population des écoles concernées.

Des entretiens avec les enseignants ont mis en évidence une difficulté à traiter la question du vocabulaire en dehors des seules activités lexicales telles qu'elles sont proposées dans les manuels, et à aucun moment en lien avec les textes à rédiger. D’après ces entretiens, les enseignants passaient environ le même temps hebdomadaire à l'enseignement du lexique.

3. Les départements géographiques dans lesquels se situent les classes sont le Val de Marne et la Seine-Saint-Denis. 
Les caractéristiques des élèves sont présentées dans le Tableau 1.

Tableau 1 : Caractéristiques des élèves

\begin{tabular}{|l|l|l|l|l|}
\hline & Nombre & Âge moyen & Genre \\
\cline { 4 - 5 } & & & Filles & Garçons \\
\hline Groupe témoin & 62 & 10,7 ans & 32 & 30 \\
\hline Groupe expérimental & 88 & 10,8 ans & 45 & 43 \\
\hline Total & 150 & & 77 & 73 \\
\hline
\end{tabular}

\section{Matériel et procédure}

Le matériel consiste, pour chacune des cinq séquences, en un texte de littérature de jeunesse «lanceur » de l'activité d'écriture et une fiche de ressources thématiques et narratologiques, soit cinq textes et cinq fiches de ressources pour l'ensemble du dispositif.

Les cinq séquences d'écriture incluent le prétest (Séance 1) et le posttest (Séance 5). Les différents textes « lanceurs » ont porté sur des thématiques familières à la littérature de jeunesse, ancrées dans le patrimoine culturel et littéraire (thème de la solitude, de l'amitié, de la ruse, de la tromperie, des métamorphoses). Les thèmes choisis correspondaient, d'une part, à une progressivité en fonction de la difficulté d'accès des textes, et d'autre part, à la possibilité de réemploi du vocabulaire d'une séquence d'enseignement à la suivante, soit par stricte réutilisation, soit par transposition des unités lexicales ou des usages métaphoriques.

Le protocole expérimental a consisté en la rédaction de cinq suites de texte régulièrement réparties dans l'année scolaire : (i) un prétest d'écriture en septembre; (ii) trois séquences d'apprentissage en novembre, janvier, mars; (iii) un posttest en mai. La suite de texte rédigée en posttest a donné lieu à la comparaison avec la version du prétest, dans le groupe expérimental et dans le groupe témoin.

Dans les classes témoins, les élèves ont réalisé le prétest et le posttest et les trois séquences intermédiaires sans bénéficier des fiches ressources. En revanche, les enseignants des classes expérimentales ont consacré un temps égal à celui utilisé pour le protocole avec les fiches et les textes ressources.

Lors de la séance 1, les élèves ont lu un texte court. Ensuite, l'enseignant a procédé à un questionnement sur le sens des mots difficiles. A suivi une première version d'écriture. Puis la séance 2 a proposé la lecture critique et récriture instrumentée des premières versions ainsi que la présentation d'outils d'aide à la récriture (listes de mots ou expressions fabriqués par l'enseignant, familiarisation à la récriture à partir des outils présentés, récriture par chaque élève de son texte initial).

Une grille thématique de ressources lexicales a été élaborée par l'expérimentateur en proposant des entrées grammaticales variées afin d'éviter la surreprésentation des noms. Cette grille a fourni 
des listes de noms et groupes nominaux, mais aussi d'adjectifs qualificatifs, de verbes et d'adverbes. Celles-ci favorisent les choix des mots permettant d'enrichir les productions textuelles initiales (cf. Annexe 1).

\section{Questions de recherche}

Les questions de recherche portent sur les aspects quantitatifs et qualitatifs de la production de textes réalisés par les élèves des deux groupes.

Sur le plan quantitatif, nous formulons trois hypothèses.

La première question concerne l'augmentation du volume des textes des élèves du groupe expérimental par rapport à ceux du groupe témoin, entre le prétest et le posttest. Selon notre première hypothèse, nous prédisons que les textes réalisés en posttest par les élèves du groupe expérimental indiqueront des progrès plus importants en termes de nombre de mots utilisés, par rapport au prétest, que ceux du groupe témoin.

La deuxième question porte sur un aspect plus stratégique, qui s'observe en comparant deux versions successives d'un même texte (V1 et V2), réalisées au cours d'une même semaine. Cette comparaison permet de vérifier la place des ajouts, selon le modèle princeps de la génétique textuelle (Grésillon, 1994). Il s'agit de quantifier ces ajouts d'une version à l'autre pour les élèves du groupe témoin, comme pour les élèves du groupe expérimental, en fonction de l'endroit du texte où ils sont situés, soit à la fin, soit à l'intérieur du texte initial. La deuxième hypothèse est celle-ci : les élèves ayant bénéficié du dispositif élaboré pour les besoins de la recherche ajouteront plus de mots dans le corps de leur texte écrit en version 1 que ceux du groupe témoin. Nous considérons en effet que le développement de la compétence lexicale conduit à densifier le texte initial pour en renforcer le sens, plutôt qu'à allonger ce texte par de nouvelles péripéties.

La troisième hypothèse consiste à prédire que les élèves du groupe témoin ajouteront plus de mots en fin de texte que ceux du groupe expérimental. Cette hypothèse s'appuie sur la même base de raisonnement que la précédente. Améliorer ses performances en littéracie conduit à densifier un texte plutôt qu'à augmenter sa longueur. L'augmentation des compétences lexicales va de pair avec la compétence à rédiger un texte.

Des analyses qualitatives viendront compléter les données quantitatives exposées, à l'appui des interprétations. Elles soutiendront la quatrième hypothèse prédisant la complémentarité des ressources associées au dispositif. 


\section{Résultats et analyse}

\section{Augmentation du volume des textes}

La première question concerne l'évolution des performances rédactionnelles mesurées à l'aune de l'augmentation du nombre de mots entre le début et la fin de l'année scolaire, globalement et en moyenne. Observe-t-on une évolution du volume des textes rédigés plus importante pour les élèves du groupe expérimental que pour les élèves du groupe témoin?

D’un point de vue quantitatif, la comparaison entre les textes réalisés en version 1 lors du prétest et du posttest indique une progression importante du volume, en nombre de mots rédigés, à l'avantage du groupe expérimental. Les élèves du groupe expérimental passent en moyenne d'un texte de 9701 mots à un texte de 13055 mots (cf. Tableau 2), alors que les élèves du groupe témoin progressent de 10716 mots à 12341 mots (cf. Tableau 2), entre le texte du prétest et le texte du posttest.

L'augmentation totale du nombre de mots est ainsi de + 3354 mots pour le groupe expérimental vs +1625 mots pour le groupe témoin. En moyenne, elle est de 38,11 mots par élève du groupe expérimental vs + 26,21 mots par élève du groupe témoin (cf. Tableau 2).

Tableau 2 : Évolution des textes en nombre de mots, du prétest au posttest

\begin{tabular}{|l|l|l|l|l|l|}
\hline & Prétest & Posttest & Évolution & $\begin{array}{l}\text { Évolution moyenne } \\
\text { par élève }\end{array}$ & Écart type \\
\hline G1 témoin & 10716 & 12341 & +1625 & $+26,2$ & 14,8 \\
\hline G2 expérimental & 9701 & 13055 & +3354 & $+38,1$ & 22,1 \\
\hline
\end{tabular}

Les résultats sont significatifs. En effet, nous avons vérifié que les distributions suivent bien une loi binomiale (test de Shapiro-Wilk), avant d'appliquer le test Student, lequel permet d'affirmer que les moyennes obtenues pour l'évolution du nombre de mots des textes du groupe expérimental et du groupe témoin sont significativement différentes ( $p$-value =0,0003).

L'accroissement significativement plus important du nombre de mots dans le groupe expérimental entre prétest (réalisé lors de la séance S14) et posttest (réalisé lors de la séance S5) indique un premier effet positif du dispositif didactique mis en place. Notre première hypothèse est validée.

Comment interpréter ce résultat? Les ressources mises à disposition des élèves du groupe expérimental l'ont été dans une perspective de développement de leurs compétences lexicales et élaborées en reprenant plusieurs axes de structuration du vocabulaire. Ceux-ci ont porté sur la structuration des unités sémantiques en catégories grammaticales, en réseaux de synonymie associant unités lexicales minimales, expressions et unités sémantiques complexes. Le recours au sens propre et au sens

4. S1 = séance 1 . 
figuré, la présentation de ces ressources en langue et en discours a conduit à leur complémentarité qui a permis un apprentissage par imprégnation et mémorisation.

\section{Localisation des ajouts de l'écriture à la réécriture d'un texte}

Cet effet d'amplification des textes, particulièrement notable pour les élèves du groupe expérimental, reste à être analysé plus précisément pour caractériser plus finement l'évolution de l'écriture, ce que ne permet pas la seule comparaison de ces tests réalisés en début, puis en fin d'année.

Du point de vue de la pertinence des ajouts. Cette caractéristique des textes s'appréhende plus aisément lorsque l'on examine l'évolution entre deux versions d'un même texte, effectuées au cours d'une même semaine. Aussi nos observations portent-elles dans un deuxième temps sur une autre phase du dispositif, qui concerne les séances d'apprentissage S2, S3, S4 au cours desquelles les élèves sont invités à rédiger un premier texte, puis à le réviser après avoir pris connaissance des ressources lexicales mises à leur disposition. Notre hypothèse porte sur la place des mots ajoutés dans le texte révisé. En effet, la maîtrise de l'écriture et les opérations mentales mobilisées par la révision de textes supposent une capacité à se glisser dans le cours et le décours du texte premier pour travailler le matériau lexical et linguistique.

\section{Localisation des ajouts en fin de texte}

Dans le cadre de cet article, nous avons ciblé nos observations sur la séance 4, qui a eu lieu à la fin d'avril, six semaines avant le posttest. À ce moment de l'année scolaire, les élèves du groupe expérimental ont la pleine maîtrise des ressources mises régulièrement à leur disposition.

Dans les textes des élèves du groupe témoin, les ajouts effectués à partir de la version 1 se trouvent massivement placés en fin de texte (1 573 mots sur les 1854 mots globalement ajoutés), soit en moyenne 25,4 mots par élève en fin de texte sur la moyenne de 29,9 mots par élève (cf. Tableau 3) pour l'ensemble des textes.

La comparaison entre le nombre moyen d'ajouts en fin de texte pour les deux groupes (25,37 pour le groupe témoin G1 vs 14,62 pour le groupe expérimental G2) (cf. Tableau 3) indique que celui-ci ne recourt pas abondamment à ce mode de spatialisation textuelle de l'ajout. 
Tableau 3 : Nombre et types d'ajouts effectués sur la version 2 de la séquence 4

\begin{tabular}{|l|l|l|l|l|l|l|}
\hline & \multicolumn{2}{|l|}{ Version 2 } \\
& Fin du texte & $\begin{array}{l}\text { Moyenne par } \\
\text { élève }\end{array}$ & $\begin{array}{l}\text { Intérieur du } \\
\text { texte }\end{array}$ & $\begin{array}{l}\text { Moyennepar } \\
\text { élève }\end{array}$ & Total & $\begin{array}{l}\text { Moyennepar } \\
\text { élève }\end{array}$ \\
\hline G1 témoin & 1573 & 25,4 & 281 & 4,5 & 1854 & 29,90 \\
\hline G2 expérimental & 1287 & 14,6 & 3032 & 34,5 & 4319 & 49,07 \\
\hline
\end{tabular}

Pour la comparaison des mots ajoutés en fin de texte, le recours aux "diagrammes en boîte », ou boîtes de Tukey, permet de vérifier que la majorité des élèves du groupe témoin se situe au-dessus de la moyenne du groupe expérimental, avec une médiane $(25,4)$, au-dessus du troisième quartile du groupe expérimental (cf. Figure 1). L'hypothèse 2 est validée: les élèves du groupe témoin concentrent leurs ajouts à la fin du texte qu'ils ont pour consigne de réviser dans son intégralité, témoignant ainsi de leur difficulté à réélaborer leur texte ou à l'enrichir. Les ajouts terminaux constituent soit une conclusion auparavant absente, soit une relance du texte et un nouvel épisode, tous deux en décalage avec le but visé par l'activité et sa description.

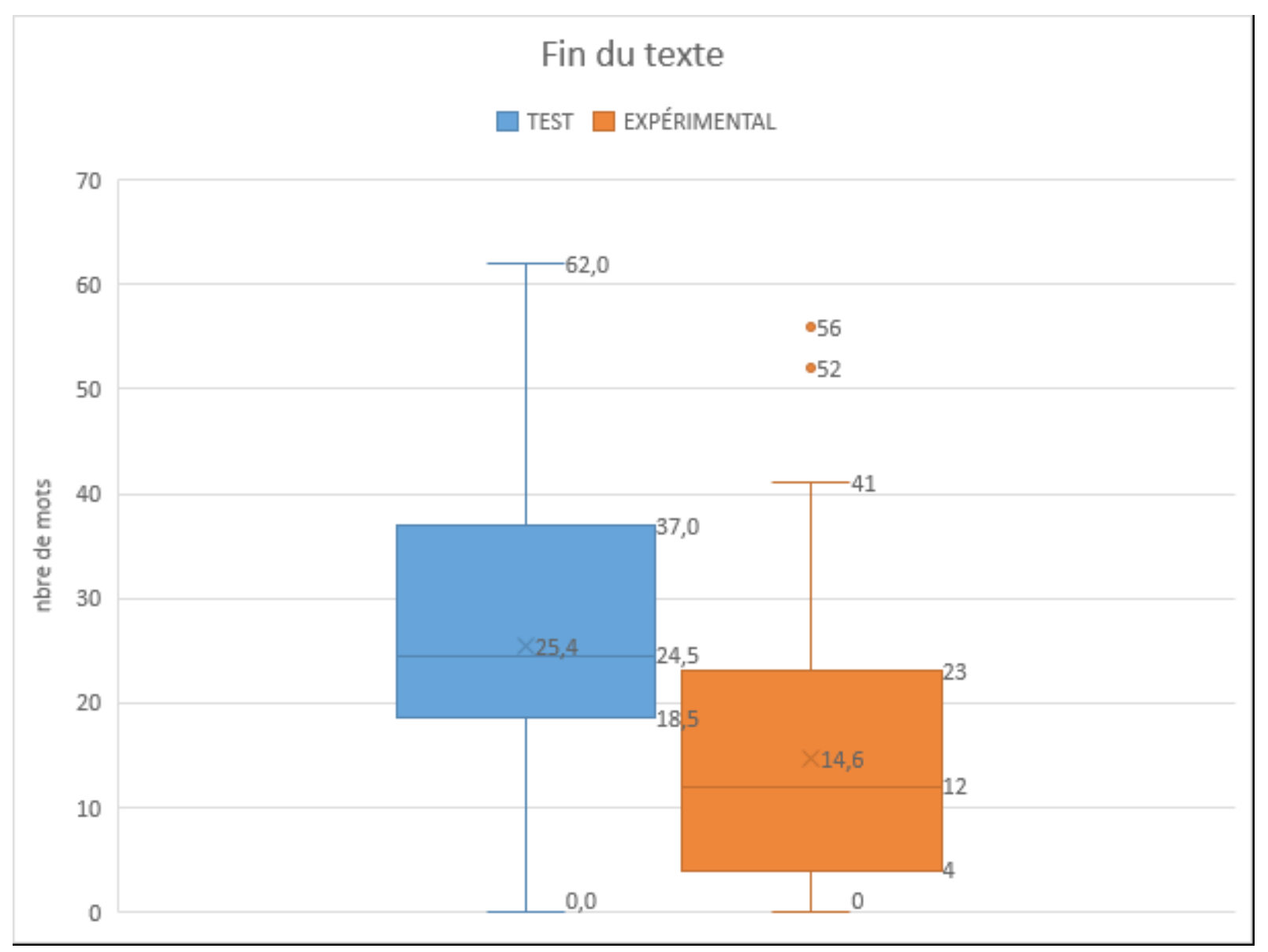

Figure 1 : Nombre de mots ajoutés en fin de texte entre la version 1 et la version 2 
La place des ajouts rend compte de la pertinence de l'activité de révision et de l'usage raisonné du lexique en vue d'améliorer la première version d'écriture.

La comparaison des versions 2 des textes du groupe témoin et du groupe expérimental indique des pratiques différentes en termes de révision. En contrepoint des ajouts en fin de texte, certains élèves recourent à une répartition des mots ajoutés à différents endroits du texte, au sein même des phrases ou constituent des incises interphrastiques complètes. Les résultats obtenus s'avèrent très différents des conclusions précédentes lorsque l'on s'intéresse à l'évolution interne au texte initial, tel qu'il a été rédigé en version 1. En effet, si les élèves du groupe expérimental ajoutent peu de mots à la fin de leur texte écrit en version 1, soit en moyenne 14,62 mots sur un total moyen d'ajouts de 49,07 mots (cf. Tableau 3), ils en ajoutent bien davantage au fil des phrases, avec un score de + 34,45 mots (cf. Tableau 3).

La localisation des mots ajoutés à l'intérieur du texte est un indicateur de la capacité à améliorer un texte et à en préciser les unités sémantiques. Ainsi, le groupe expérimental G2 compte un nombre d'ajouts à l'intérieur du texte égal à 3012 mots, alors que ces ajouts ne concernent que 281 mots dans le groupe témoin (cf. Tableau 3). Les moyennes des mots ajoutés par élève atteignent ainsi 34,5 mots pour le groupe G2 et seulement 4,5 mots pour le groupe G1. Les deux boîtes de Tukey représentant les scores des groupes G1 et G2 sont totalement disjointes (cf. Figure 2). La troisième hypothèse est validée. 


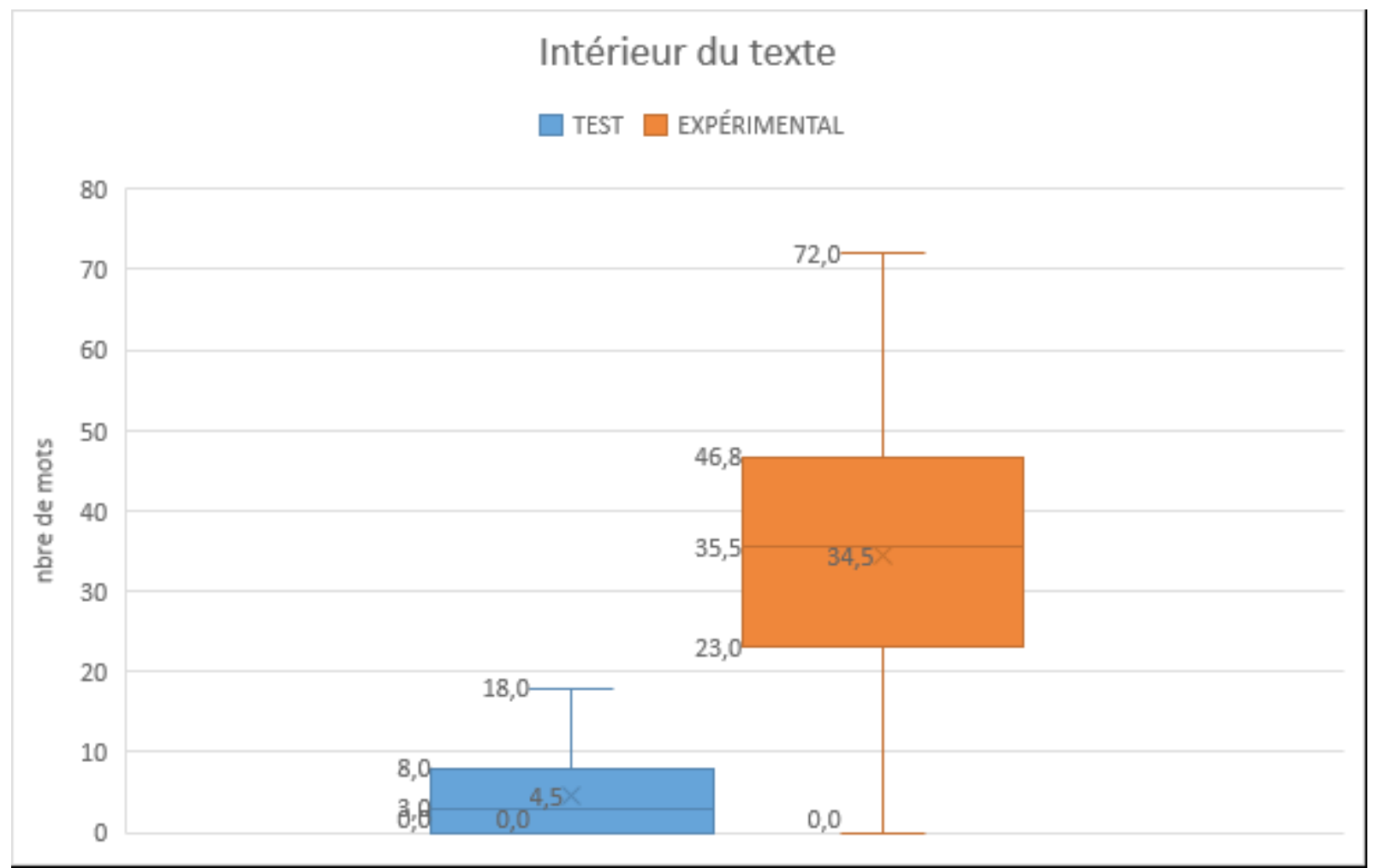

Figure 2 : Nombre de mots ajoutés à l'intérieur du texte entre la version 1 et la version 2

Par ailleurs, nous observons que le nombre total de mots des textes de la séquence 4 est supérieur à celui de la séquence 5, correspondant au posttest. Ce résultat peut être interprété comme une légère régression entre la séquence 4 et la séquence 5, laquelle est cependant nuancée par une progression linéaire assurée tout au long de l’année scolaire.

\section{Éléments d'analyse qualitative}

Nous prenons pour illustration l'analyse d'un texte portant sur la thématique des métamorphoses et rédigé par un élève appartenant au groupe expérimental. Nous nous appuyons sur les ajouts effectués dans la deuxième version du texte, dont les emprunts issus du texte source, "L'Oiseau d'or »"5. Parmi les ressources lexicales mises à disposition des élèves de ce groupe, a été proposée une fiche de vocabulaire thématique présentée par catégories narratologiques et grammaticales (cf. Annexe 1). Cette fiche de ressources lexicales a été élaborée par les expérimentateurs en proposant des entrées grammaticales diversifiées afin d'éviter la surreprésentation du nom (Nonnon, 2011). D'autres outils complémentaires d'aide à la récriture sont fournis tels une liste d'expressions (cf. Annexe 2),

5. Mouloud Mammeri, Contes berbères de Kabylie, Pocket Jeunesse, 2000. 
de synonymes (cf. Annexe 3) et un extrait de roman de littérature de jeunesse portant sur la même thématique 6 .

L'ensemble de ces ressources favorise le choix des mots permettant d'enrichir les productions textuelles initiales et de produire des significations au-delà de la dimension encyclopédique des ajouts, comme le montre le début de ce texte d’Arthur , élève du groupe expérimental, dans sa version révisée :

Aziza traverse le désert et trouva un scorpion qui essayait de la piquer. Elle le transperça avec son couteau. Après alla dans la forêt et trouva un groupe de loups qui essayaient de la mordre, ensuite elle vit l'oiseau d'or. II était bien doré. Il était aussi mélodieux et chatoyant mais très maléfique et très attirant. Ensuite elle aperçut son frère, elle était désespérée. Elle ne pouvait rien faire, alors elle fit un piège, captura l'oiseau d'or et obligea l'oiseau à sauver son frère. [... $]^{8}$

Cet exemple illustre un effet du dispositif d'aide lexicale qui corrobore nos hypothèses. L'enrichissement du vocabulaire en langue et en discours se fonde sur la mobilisation des différentes ressources lexicales dont certaines sont conjuguées. Ainsi, parmi les aides utilisées par Arthur, figure le recours aux adjectifs qualificatifs de la fiche (cf. Annexe 1), avec l'usage des adjectifs connotant simultanément la séduction ( "mélodieux », «chatoyant»), et le péril (« maléfique ») qui caractérisent l'oiseau d'or. Ces adjectifs deviennent ainsi des catalyseurs de sens et permettent l'embrayage du récit avec des chaînes de causalité. Les intentions et les chaînes de causalité apparaissent bien organisées dans ce récit. Le péril à croiser le chemin de l'oiseau est mis en scène. L'usage des verbes « vit », puis « aperçut » orchestre une autre scénarisation où l'oiseau apparaît au premier plan dans ses mouvements, tandis que sa victime, le frère d’Aziza, n'est qu'aperçu, en arrière-plan, dans sa position de victime pétrifiée, ce qui déclenche le plan d'action de la protagoniste pour délivrer son frère. Une autre catégorie d'ajouts relève du discours de l'enseignant, qui, après la lecture du texte « lanceur », avait recentré l'écoute des élèves sur la mémorisation et l'appropriation du vocabulaire. Usant de paraphrases favorisant l'encodage (Jousse et al., 2008), elle avait focalisé l'attention sur plusieurs mots du texte «lanceur » à forte charge sémantique. La glose des mots « attirant », " désespérée », «transperça » en a ainsi permis la mémorisation et le réinvestissement en discours.

La complémentarité des ressources lexicales mobilisées permet de valider notre quatrième hypothèse. En effet, dans le groupe expérimental la structuration du vocabulaire par la catégorisation des entités grammaticales et narratologiques et le travail sur les diverses modalités d'encodage, dont l'usage de la paraphrase, ont permis de diversifier les modes de désignation. Cependant, comme le

6. Anthony Browne, La Forêt profonde, L'école des loisirs, 2006.

7. Afin de préserver l'anonymat, le prénom de l'auteur a été modifié.

8. L'extrait que nous en présentons du texte d'Arthur contient 84 mots, sur les 163 mots qui composent cette version révisée dans son ensemble. Il s'agit ainsi d'un texte dont la longueur se situe dans la moyenne des productions écrites du groupe expérimental auquel il appartient. 
montre le texte d'Arthur, choisi à titre d'exemple, les ressources lexicales construites par les élèves leur ont permis d'accéder à l'élaboration de la référence et de la signification (Nonnon, 2012).

\section{Discussion}

La complexité de l'analyse des résultats tient à la complexité même du contexte de l'enseignement qui se situe au centre de trois niveaux de contexte, le microcontexte, le contexte médian et le macrocontexte (Sauvage-Luntade et Tupin, 2012). Le microcontexte concerne les caractéristiques des élèves, de la classe, de l'établissement', les contenus disciplinaires, notamment; le contexte médian est celui des directives institutionnelles, des programmes, des décisions collégiales du travail collaboratif; enfin le macrocontexte concerne les politiques éducatives, pédagogiques,et de formation des enseignants. C'est pourquoi les précautions méthodologiques ont consisté à contrôler dans la mesure du possible ces différents facteurs avant de nous intéresser au microcontexte que constitue le dispositif didactique mis en place.

Globalement, les résultats montrent que ce dispositif a permis aux élèves d'améliorer leur performances en littéracie en produisant des textes plus longs (cf. Tableau 2) et plus complets. II convient cependant de considérer la qualité des textes produits à partir de critères habituels qui restent à rappeler (pertinence, cohérence, enchaînements interphrastiques, etc.), laquelle a progressé à l'aune des insertions de mots nouveaux et d'un registre littéraire affirmé.

Observons plus précisément comment se jouent les modifications entre des textes comparables avec les versions 1 et 2 d'un même texte. La comparaison de ces versions se prête en effet à un examen attentif de l'effet immédiat du dispositif d'apprentissage. Cette observation montre l'emploi de termes synonymes avec des gradations, et des caractérisations plus précises des personnages, de leurs intentions qui motivent leurs actions et donnent de la cohérence aux verbes d'action. Les récits sont ainsi mieux construits. II s'agit là d'un résultat à court terme dont nous pouvons mesurer les effets à plus long terme en décrivant les différences d'évolution.

Certes, il est toujours difficile de mesurer précisément les effets d'un dispositif en contexte écologique, dans la mesure où il semble impossible de contrôler tous les paramètres de la situation d'apprentissage. Des biais sont possibles, qui peuvent conduire à nuancer les résultats et leur interprétation. Par exemple, le vocabulaire utilisé dans d'autres disciplines autour de thématiques traitées de manière interdisciplinaire peut jouer un rôle important dans la mémorisation et l'appropriation de certains mots réutilisés plus aisément par les élèves. Lors de l'expérimentation menée, nous nous sommes assurées de conditions identiques de traitement des thèmes dans chacune des classes, expérimentales et témoins. Le choix des thèmes l'a été également en raison de l'absence de porosité entre ce vocabulaire thématique et le réemploi dans d'autres disciplines, afin de limiter le plus possible l'étude aux effets directs du dispositif.

9. Situation géographique, milieu social des familles, catégorie REP+... 
Cette étude constitue un exemple de recherche impliquée, où, par cette implication, les différents partenaires de la communauté enseignante, chercheurs et enseignants ont pu prendre en compte une question professionnelle : comment enrichir le lexique des élèves pour leur permettre d'écrire des textes narratifs plus cohérents et plus denses? Cette question a été retravaillée en termes d'hypothèses de recherche: Quel est l'effet de dispositifs d'acquisition lexicale sur le réinvestissement dans l'écriture, lorsque ces dispositifs fournissent des aides thématiques et sémantiques ciblées? Quel est leur effet à court terme ? À plus long terme ? Quel lien existe-t-il entre la possibilité donnée d'emprunter un mot qui caractérise les personnages et la création par l'élève d'une expansion narrative donnant lieu à un développement nouveau et inattendu ? Comment le progrès linguistique permet-il d'accéder à une dimension langagière?

Certes, les supports d'apprentissage lexical décrits contribuent, de diverses manières et à différents niveaux, à l'acquisition de compétences narratives. Cependant, il importe de souligner l'importance de la lecture en réseau, qui constitue une source de découverte et de réinvestissement de mots d'un même domaine référentiel.

La circulation des mots entre l'oral et l'écrit permet d'activer et de dynamiser l'usage du vocabulaire dans les différentes composantes de l'enseignement du français où dire, lire et écrire se font écho. Les contraintes de l'activité enseignante, y compris celle de la répartition du temps entre apprentissage et évaluation de ces apprentissages, laissent une place décisive à la manière de mettre en place des situations de régulation, qu'elles soient immédiates ou différées (Bru, 2014). C'est là que peuvent se jouer les effets « retombées » de la recherche.

Des effets liés à la motivation ont pu jouer un rôle important dont il est difficile de mesurer l'impact. Les textes rédigés par les élèves ont été mis en ligne sur un site web qui a permis de les diffuser largement et de les faire ainsi accéder à un lectorat plus vaste que celui ordinairement constitué par le seul enseignant de la classe. Ce facteur a pu catalyser la mobilisation des élèves dans l'activité rédactionnelle et contribuer au maintien de leur attention au fil des séquences d'apprentissage. Le caractère d'auctorialité conféré par la publication électronique a été pour certains une source d'engouement pour l'écriture, comme l'ont montré leurs commentaires lors des entretiens menés avec eux par les chercheurs. Cependant, la même possibilité a été offerte aux élèves du groupe témoin, ce qui permet de pondérer l'effet de ce facteur « publicisation de l'écriture ».

Dans le groupe expérimental, le mot emprunté est devenu un noyau sémantique autour duquel se sont greffés des éléments phrastiques et des relations de causalité, le plus souvent intentionnelle (Searle, 2012). On constate ainsi que les élèves en difficulté puisent dans le répertoire lexical de l'enseignant ou des grilles à disposition, alors que les élèves les plus performants s'inspirent des textes d'auteurs. 
Cette étude portant sur la partie du corpus constitué par les écrits et les récritures des élèves de troisième année permet de présenter les résultats de scripteurs novices. Ce n'est qu'un volet de l'étude qui concerne l'ensemble du cycle trois du système scolaire français ${ }^{10}$ pour lequel les données sont en cours de traitement. Vérifier si les premiers résultats obtenus pour la classe de troisième année concordent avec ceux obtenus pour la quatrième, la cinquième année et l'ensemble du cycle trois permettra de consolider les résultats et leurs interprétations.

Par ailleurs, plusieurs dispositifs d'aide lexicale ont été testés dans la recherche, qui permettront de comparer leurs effets respectifs en fonction des différents profils d'élèves pour élaborer de nouveaux modèles de ressources lexicales au service de l'écrit narratif en vue d'irriguer les pratiques enseignantes.

\section{Remerciements}

Nous remercions les enseignants de l'ensemble des classes expérimentales et des classes témoins, qui ont accepté de participer à cette expérimentation et aux entretiens qui ont suivi.

10. Au moment où ont été recueillies les données, le cycle trois de l'école élémentaire française comprend le cours élémentaire deuxième année, le cours moyen première année et le cours moyen deuxième année. 


\section{Bibliographie}

Barton, J. et Hamilton, M. (2010). La littéracie, une pratique sociale. New Literacy Studies, Langage et société, 133, 4562.

Bru, M. (2014). Le choix de l'observation pour l'étude des pratiques enseignantes. Recherches en éducation, 19, 7-17.

Bucheton, D. (2014). Refonder l'enseignement de l'écriture. Vers des gestes professionnels plus ajustés. Paris, Retz.

Colé, P. (2011). Le développement du vocabulaire à l'école primaire : Les apports de la dimension morphologique de la langue. L’Acquisition du vocabulaire, Paris, Ministère de l'Éducation nationale - Éduscol. http://imagesetlangages.fr/PE2/docs application/Developpementvoc ecprimaire.pdf

Crinon, J., Marin, B. et Lallias, J.-C. (2006). Enseigner la littérature au cycle trois. Paris, Nathan (collection Les repères pédagogiques).

Dechamboux, L. et Mottier-Lopez, L. (2015). L'enquête de l'enseignant-chercheur et du chercheur : l'abduction au cœur du processus et quelques raisons de les comparer. Recherches qualitatives, 34, 173-195.

Denhière, G. et Jhean-Larose, S. (2011). L'Acquisition du vocabulaire, Paris, Ministère de l'Éducation nationale - Éduscol. http://sip1d.ac-mayotte.fr/IMG/pdf/Guy Denhiere 111202 avec couv 201142.pdf

Ducan, L., Colé, P. et Casalis, S. (2009). Early metalinguistic awareness of derivational morphology: Observations from a comparison of English and French. Applied Psycholinguistics, 30, 405-440.

Garcia-Debanc, C., Duvignau, K, Dutrait, C. et Gangneux, M. (2009). Enseignement du lexique et production écrite. Un travail sur les verbes de déplacement à la fin de l'école primaire. Pratiques, 141-142, 208-232. Numéro thématique «La synonymie ».

Grésillon, A. (1994). Éléments de critique génétique. Lire les manuscrits modernes, Paris, Presses universitaires de France.

Jousse, A.-L., Polguère, A. et Tremblay, O. (2008). Du dictionnaire au site lexical pour l'enseignement/ apprentissage du vocabulaire. Observatoire de Linguistique Sens-Texte. Université de Montréal.

Kleiber, G. (2009). La synonymie-« identité de sens » n'est pas un mythe. Pratiques, 141-142, 9-25. Numéro thématique « La synonymie».

Lee, J. (2011). Size matters: Early vocabulary as a predictor of language and literacy competence. Applied Psycholinguistics, 32(1), 69-92.

Marin, B. (2013). «Quand les savoirs font autorité : langage et métalangage », Recherche et Formation, $71,4860$. 
McCutchen, D. (2011). From novice to expert: implications of language skills and writing-relevant knowledge for memory during the development of writing skill. Journal of Writing Research, 3(1), 51-68.

Nonnon, É. (2011). Quelques critères pour le développement du vocabulaire. Paris, Ministère de l'Éducation nationale - Éduscol. http://www21.ac-lyon.fr/etab/ien/rhone/lyonstefoy/IMG/pdf/ Elisabeth Nonnon 111209 C 201951.pdf

Nonnon, É. (2012). La didactique du français et l'enseignement du vocabulaire, dans vingt ans de revues de didactique du français langue première, Repères, 46, 33-73. Vingt ans de recherches en didactique du français (1990-2010).

Reed, D. K. (2008). A synthesis of morphology interventions and effects of reading outcomes for students in grades K-12. Learning Disabilities Research \& Practice, 23(1), 36-49.

Rosenthal, J. et Ehri, L. C. (2011). Pronouncing new words aloud during the silent reading of text enhances fifth graders' memory for vocabulary words and their spellings. Reading and Writing, 24(8), 921-950.

Sardier, A. (2012). Favoriser l'accès lexical en situation de production écrite. Pratiques, 155-156, $127-$ 146. Numéro thématique « Lexique et écriture ».

Sardier, A. et Grossmann, F. (2010). Comment favoriser le réemploi lexical ? Lexique Vocabulaire Recherches, 53, 9-29.

Sauvage-Luntade, L. et Tupin, F. (2012). La compétence de contextualisation au cœur de la SAF. Revue Phronesis, 1(1), 102-117.

Searle, J. (2012). Qu'est-ce que le langage ? Pratiques, 155-156, 228-250. Numéro thématique « Lexique et écriture».

Verhoeven, L. (2011). Vocabulary growth and reading development across the elementary school years. Scientific Studies of Reading, 15(1), 8-25.

Wanlin, P. et Crahay, M. (2012). La pensée des enseignants pendant l'interaction en classe. Une revue de la littérature anglophone. Éducation et Didactique, 1(6), 9-46. 


\section{Annexes}

\section{Annexe $1:$ Ressources thématiques et narratologiques}

\begin{tabular}{|c|c|c|c|c|}
\hline & Le frère & La sœur & L'oiseau d'or & Les dangers rencontrés \\
\hline Noms GN & $\begin{array}{l}\text { le malheureux (garçon), le frère } \\
\text { attentionné, le frère d’Aziza, ce } \\
\text { garçon généreux }\end{array}$ & $\begin{array}{l}\text { la fille exigeante, la petite fille } \\
\text { tyrannique, la sœur d'Aziz, } \\
\text { sanglots déchirants }\end{array}$ & $\begin{array}{l}\text { l'oiseau mélodieux, l'oiseau } \\
\text { maléfique, } \\
\text { le plumage chatoyant, le chant } \\
\text { envoûtant }\end{array}$ & $\begin{array}{l}\text { le marécage, le piège, la tem- } \\
\text { pête, le serpent, le scorpion } \\
\text { menaçant, la chaleur torride, } \\
\text { le soleil de plomb }\end{array}$ \\
\hline Adjectifs & $\begin{array}{l}\text { courageux, distrait, inattentif, } \\
\text { pétrifié, figé }\end{array}$ & $\begin{array}{l}\text { capricieuse, téméraire, affec- } \\
\text { tueuse, volontaire }\end{array}$ & $\begin{array}{l}\text { rusé, insistant, opiniâtre, } \\
\text { persévérant }\end{array}$ & \\
\hline Adverbes & $\begin{array}{l}\text { spontanément, affectueuse- } \\
\text { ment, malheureusement }\end{array}$ & prestement & $\begin{array}{l}\text { mélodieusement, sournoise- } \\
\text { ment }\end{array}$ & \\
\hline Verbes & s'anime, embrasse & $\begin{array}{l}\text { parcourt, rencontre, aperçoit, } \\
\text { craint }\end{array}$ & $\begin{array}{l}\text { chante, questionne, fige, } \\
\text { transforme, } \\
\text { ricane, volète }\end{array}$ & \\
\hline
\end{tabular}

\section{Annexe 2 : Liste d'expressions}

N'écoutant que son bon cœur - reprend vie - reprend des couleurs - bondit de joie - à pas feutrés

- fier comme un paon - folle de joie - plus fort que la mort - pleure de joie - presser le pas - être au bord des larmes - gagné(e) par la peur.

\section{Annexe 3 : Liste de synonymes}

- dire : demander, questionner, répondre, s'écrier, rétorquer

- $\quad$ aller : s’enfoncer, pénétrer, progresser, poursuivre, accélérer

- $\quad$ médusé : surpris, étonné, abasourdi, admiratif, pétrifié, figé, immobile, statufié 